\title{
DIFFERENTIAL SANDWICH THEOREMS FOR SOME SUBCLASS OF ANALYTIC FUNCTIONS ASSOCIATED WITH LINEAR OPERATORS
}

\author{
T.N. Shanmugam, M.P. Jeyaraman and A. Singaravelu
}

\begin{abstract}
Let $q_{1}$ and $q_{2}$ be univalent in $\Delta:=\{z:|z|<1\}$ with $q_{1}(0)=q_{2}(0)=1$. We give some applications of first order differential subordination and superordination to obtain sufficient conditions for a normalized analytic functions $f$ with $f(0)=0$, $f^{\prime}(0)=1$ to satisfy

$$
q_{1}(z) \prec{\frac{z f^{\prime}(z)}{f(z)}}^{\lambda} \prec q_{2}(z) .
$$
\end{abstract}

\section{INTRODUCTION}

Let $\mathcal{H}$ be the class of functions analytic in $\Delta:=\{z:|z|<1\}$ and $\mathcal{H}[a, n]$ be the subclass of $\mathcal{H}$ consisting of functions of the form $f(z)=a+a_{n} z^{n}+$ $a_{n+1} z^{n+1}+\cdots$. Let $\mathcal{A}$ be the subclass of $\mathcal{H}$ consisting of functions of the form $f(z)=z+a_{2} z^{2}+\cdots$. Let $p, h \in \mathcal{H}$ and let $\phi(r, s, t ; z): \mathbb{C}^{3} \times \Delta \rightarrow \mathbb{C}$. If $p$ and $\phi\left(p(z), z p^{\prime}(z), z^{2} p^{\prime \prime}(z) ; z\right)$ are univalent and if $p$ satisfies the second order superordination

$$
h(z) \prec \phi\left(p(z), z p^{\prime}(z), z^{2} p^{\prime \prime}(z) ; z\right),
$$

then $p$ is a solution of the differential superordination (1). (If $f$ is subordinate to $F$, then $F$ is called a superordinate of $f$.) An analytic function $q$ is called a subordinant if $q \prec p$ for all $p$ satisfying (1). An univalent subordinant $\bar{q}$ that satisfies $q \prec \bar{q}$ for all subordinants $q$ of (1) is said to be the best subordinant. Recently Miller and

Received 6 February 2006, revised 25 October 2006, accepted 15 November 2006 .

2000 Mathematics Subject Classification: Primary 30C45, Secondary 30C 80.

Key words and Phrases: Differential Subordination, Differential Superordination, Subordinant. 
Mocanu [10] obtained conditions on $h, q$ and $\phi$ for which the following implication holds:

$$
h(z) \prec \phi\left(p(z), z p^{\prime}(z), z^{2} p^{\prime \prime}(z) ; z\right) \Rightarrow q(z) \prec p(z) .
$$

Using the results of Miller and Mocanu [10], Bulboaca [3] considered certain classes of first order differential superordinations as well as superordination-preserving operators [2]. Using the results of [3], Shanmugam et al. [12] obtained sufficient conditions for a normalized analytic function $f(z)$ to satisfy

$$
q_{1}(z) \prec \frac{f(z)}{z f^{\prime}(z)} \prec q_{2}(z)
$$

and

$$
q_{1}(z) \prec \frac{z^{2} f^{\prime}(z)}{\{f(z)\}^{2}} \prec q_{2}(z)
$$

respectively where $q_{1}$ and $q_{2}$ are given univalent functions in $\Delta$.

For $\alpha_{j} \in \mathbb{C} \quad(j=1,2, \ldots, l)$ and $\left.\beta_{j} \in \mathbb{C} \backslash \mathbb{Z}_{0}^{-}:=\{0,-1,-2, \ldots\}, j=1,2, \ldots m\right)$, the generalized hypergeometric function ${ }_{l} F_{m}\left(\alpha_{1}, \ldots, \alpha_{l} ; \beta_{1}, \ldots, \beta_{m} ; z\right)$ is defined by the infinite series

$$
\begin{gathered}
{ }_{l} F_{m}\left(\alpha_{1}, \ldots, \alpha_{l} ; \beta_{1}, \ldots, \beta_{m} ; z\right):=\sum_{n=0}^{\infty} \frac{\left(\alpha_{1}\right)_{n} \ldots\left(\alpha_{l}\right)_{n}}{\left(\beta_{1}\right)_{n} \ldots\left(\beta_{m}\right)_{n}} \frac{z^{n}}{n !} . \\
\left(l \leq m+1 ; l, m \in \mathbb{N}_{0}:=\mathbb{N} \cup\{0\}\right),
\end{gathered}
$$

where $(a)_{n}$ is the Pochhammer symbol defined by

$$
(a)_{n}:=\frac{\Gamma(a+n)}{\Gamma(a)}= \begin{cases}1, & (n=0) \\ a(a+1)(a+2) \ldots(a+n-1), & (n \in \mathbb{N}) .\end{cases}
$$

Corresponding to the function

$$
h\left(\alpha_{1}, \ldots, \alpha_{l} ; \beta_{1}, \ldots, \beta_{m} ; z\right):=z_{l} F_{m}\left(\alpha_{1}, \ldots, \alpha_{l} ; \beta_{1}, \ldots, \beta_{m} ; z\right),
$$

the Dziok-Srivastava operator [5] (see also [13]) $H_{m}^{l}\left(\alpha_{1}, \ldots, \alpha_{l} ; \beta_{1}, \ldots, \beta_{m} ; z\right)$ is defined by the Hadamard product

$$
\begin{aligned}
H_{m}^{l}\left(\alpha_{1}, \ldots, \alpha_{l} ; \beta_{1}, \ldots, \beta_{m} ; z\right) f(z):= & h\left(\alpha_{1}, \ldots, \alpha_{l} ; \beta_{1}, \ldots, \beta_{m} ; z\right) * f(z) \\
& =z+\sum_{n=2}^{\infty} \frac{\left(\alpha_{1}\right)_{n-1} \ldots\left(\alpha_{l}\right)_{n-1}}{\left(\beta_{1}\right)_{n-1} \ldots\left(\beta_{m}\right)_{n-1}} \frac{a_{n} z^{n}}{(n-1) !} .(2)
\end{aligned}
$$

It is well known [5] that

$$
\begin{aligned}
\alpha_{1} H_{m}^{l} & \left(\alpha_{1}+1, \ldots, \alpha_{l} ; \beta_{1}, \ldots, \beta_{m} ; z\right) f(z) \\
= & z\left[H_{m}^{l}\left(\alpha_{1}, \ldots, \alpha_{l} ; \beta_{1}, \ldots, \beta_{m} ; z\right) f(z)\right]^{\prime} \\
& +\left(\alpha_{1}-1\right) H_{m}^{l}\left(\alpha_{1}, \ldots, \alpha_{l} ; \beta_{1}, \ldots, \beta_{m} ; z\right) f(z) .
\end{aligned}
$$


To make the notation simple, we write

$$
H_{m}^{l}\left[\alpha_{1}\right] f(z):=H_{m}^{l}\left(\alpha_{1}, \ldots, \alpha_{l} ; \beta_{1}, \ldots, \beta_{m} ; z\right) f(z) .
$$

Special cases of the Dziok-Srivastava linear operator includes the Hohlov linear operator [6] , the Carlson-Shaffer linear operator[4], the Ruscheweyh derivative operator [11], the generalized Bernardi-Libera-Livingston linear integral operator (cf. [1], [7], [8]).

\section{PRELIMINARIES}

In our present investigation, we shall need the following definition and results. In this paper unless otherwise mentioned $\alpha$ and $\beta$ are complex numbers.

Definition 2.1: [10, Definition 2, p. 817] Let $Q$ be the set of all functions $f$ that are analytic and injective on $\bar{\Delta}-E(f)$, where

$$
E(f)=\left\{\zeta \in \partial \Delta: \lim _{z \rightarrow \zeta} f(z)=\infty\right\}
$$

and are such that $f^{\prime}(\zeta) \neq 0$ for $\zeta \in \partial \Delta-E(f)$.

Theorem 2.1 : [9, Theorem 3.4h, p. 132] Let $q$ be univalent in the unit disk $\Delta$ and $\theta$ and $\phi$ be analytic in a domain $D$ containing $q(\Delta)$ with $\phi(\omega) \neq 0$ when $\omega \in q(\Delta)$.

Set $\xi(z)=z q^{\prime}(z) \phi(q(z)), h(z)=\theta(q(z))+\xi(z)$. Suppose that,

1. $\xi(z)$ is starlike univalent in $\Delta$ and

2. $\Re \frac{z h^{\prime}(z)}{\xi(z)}>0$ for $z \in \Delta$.

If $p$ is analytic in $\Delta$ with $p(\Delta) \subseteq D$, and

$$
\theta(p(z))+z p^{\prime}(z) \phi(p(z)) \prec \theta(q(z))+z q^{\prime}(z) \phi(q(z))
$$

then $p \prec q$ and $q$ is the best dominant.

Lemma 2.1 : [12] Let $q$ be univalent in $\Delta$ with $q(0)=1$. Further assuming that

$$
\Re\left[\frac{\alpha}{\beta}+1+\frac{z q^{\prime \prime}(z)}{q^{\prime}(z)}\right]>0 .
$$

If $p$ is analytic in $\Delta$, with $p(\Delta) \subseteq D$ and

$$
\alpha p(z)+\beta z p^{\prime}(z) \prec \alpha q(z)+\beta z q^{\prime}(z)
$$


then $p \prec q$ and $q$ is the best dominant.

Theorem 2.2 : [3] Let $q$ be univalent in the unit disk $\Delta$ and $\vartheta$ and $\varphi$ be analytic in a domain $D$ containing $q(\Delta)$. Suppose that

1. $\Re\left[\frac{\vartheta^{\prime}(q(z))}{\varphi(q(z))}\right]>0$ for $z \in \Delta$, and

2. $\xi(z)=z q^{\prime}(z) \varphi(q(z))$ is starlike univalent function in $\Delta$.

If $p \in \mathcal{H}[q(0), 1] \cap Q$, with $p(\Delta) \subset D$ and $\vartheta(p(z))+z p^{\prime}(z) \varphi(p(z))$ is univalent in $\Delta$, and

$$
\vartheta(q(z))+z q^{\prime}(z) \varphi(q(z)) \prec \vartheta(p(z))+z p^{\prime}(z) \varphi(p(z)),
$$

then $q \prec p$ and $q$ is the best subordinant.

Lemma 2.2 : [12] Let $q$ be univalent in $\Delta, q(0)=1$. Further assuming that $\Re\left[\frac{\alpha}{\beta} q^{\prime}(z)\right]>0$.

If $p \in \mathcal{H}[q(0), 1] \cap Q$, and $\alpha p+\beta z p^{\prime}$ is univalent in $\Delta$, and

$$
\alpha q(z)+\beta z q^{\prime}(z) \prec \alpha p(z)+\beta z p^{\prime}(z)
$$

then $q \prec p$ and $q$ is the best subordinant.

\section{SUBORDINATION RESULTS FOR ANALYTIC FUNCTIONS}

By making use of Lemma 2.3, we prove the following results.

Theorem 3.1 : Let $q$ be univalent in $\Delta$ with $q(0)=1$ and satisfying

$$
\Re\left[\frac{\alpha}{\beta}+1+\frac{z q^{\prime \prime}(z)}{q^{\prime}(z)}\right]>0 .
$$

Let

$$
\Psi(\alpha, \beta, \lambda ; z):=\alpha\left(\frac{z f^{\prime}(z)}{f(z)}\right)^{\lambda}+\beta \lambda\left(\frac{z f^{\prime}(z)}{f(z)}\right)^{\lambda}\left\{1+\frac{z f^{\prime \prime}(z)}{f^{\prime}(z)}-\frac{z f^{\prime}(z)}{f(z)}\right\} .
$$

If $f \in \mathcal{A}$ satisfies

$$
\Psi(\alpha, \beta, \lambda ; z) \prec \alpha q(z)+\beta z q^{\prime}(z)
$$

then

$$
\left(\frac{z f^{\prime}(z)}{f(z)}\right)^{\lambda} \prec q(z)
$$

and $q$ is the best dominant. 
Proof. Define the function $p(z)$ by

$$
p(z):=\left(\frac{z f^{\prime}(z)}{f(z)}\right)^{\lambda} .
$$

Then by means of simple computation we can show that

$$
\Psi(\alpha, \beta, \lambda ; z)=\alpha p(z)+\beta z p^{\prime}(z) .
$$

Now (8) becomes

$$
\alpha p(z)+\beta z p^{\prime}(z) \prec \alpha q(z)+\beta z q^{\prime}(z)
$$

and Theorem 3.1 follows by an application of Lemma 2.1.

By taking $q(z)=\frac{1+A z}{1+B z}(-1 \leq B<A \leq 1)$ we have the following Example.

Example 3.1 : Let $q(z)=\frac{1+A z}{1+B z}(-1 \leq B<A \leq 1)$ in Theorem 3.1. Further assuming that (6) holds. If $f \in \mathcal{A}$, then

$$
\begin{gathered}
\Psi(\alpha, \beta, \lambda ; z) \prec \alpha\left(\frac{1+A z}{1+B z}\right)+\beta \frac{(A-B) z}{(1+B z)^{2}}, \\
\Rightarrow \quad\left(\frac{z f^{\prime}(z)}{f(z)}\right)^{\lambda} \prec \frac{1+A z}{1+B z},
\end{gathered}
$$

and $\frac{1+A z}{1+B z}$ is the best dominant.

Also if $q(z)=\frac{1+z}{1-z}$, then for $f \in \mathcal{A}$ we have

$$
\begin{gathered}
\Psi(\alpha, \beta, \lambda ; z) \prec \alpha\left(\frac{1+z}{1-z}\right)+\frac{2 \beta z}{(1-z)^{2}}, \\
\Rightarrow \quad\left(\frac{z f^{\prime}(z)}{f(z)}\right)^{\lambda} \prec \frac{1+z}{1-z},
\end{gathered}
$$

and $\frac{1+z}{1-z}$ is the best dominant.

\section{SUPERORDINATION RESULTS FOR ANALYTIC FUNCTIONS}

Theorem 4.1 : Let $q$ be convex univalent in $\Delta$ with $q(0)=1$. Let $f \in \mathcal{A}$, $\left(\frac{z f^{\prime}(z)}{f(z)}\right)^{\lambda} \in \mathcal{H}[1,1] \cap Q$, with

$$
\Re\left[\frac{\alpha}{\beta} q^{\prime}(z)\right]>0 .
$$


If $\Psi(\alpha, \beta, \lambda ; z)$ as defined by (7) is univalent in $\Delta$, with

$$
\alpha q(z)+\beta z q^{\prime}(z) \prec \Psi(\alpha, \beta, \lambda ; z)
$$

then

$$
q(z) \prec\left(\frac{z f^{\prime}(z)}{f(z)}\right)^{\lambda}
$$

and $q$ is the best subordinant.

Proof. Theorem 4.1 follows by an application of Lemma 2.2.

By taking $q(z)=\frac{1+A z}{1+B z}(-1 \leq B<A \leq 1)$ in Theorem 4.1, we have the following Example.

Example 4.1 : Let $q$ be convex univalent in $\Delta$.

Also let $f \in \mathcal{A},\left(\frac{z f^{\prime}(z)}{f(z)}\right)^{\lambda} \in \mathcal{H}[1,1] \cap Q$. Further assuming that (9) holds. If $\Psi(\alpha, \beta, \lambda ; z)$ as defined by $(7)$ is univalent in $\Delta$, and

$$
\alpha\left(\frac{1+A z}{1+B z}\right)+\frac{\beta(A-B) z}{(1+B z)^{2}} \prec \Psi(\alpha, \beta, \lambda ; z),
$$

then

$$
\frac{1+A z}{1+B z} \prec\left(\frac{z f^{\prime}(z)}{f(z)}\right)^{\lambda}
$$

and $\frac{1+A z}{1+B z}$ is the best subordinant.

Inparticular, we have

$$
\alpha\left(\frac{1+z}{1-z}\right)+\frac{2 \beta z}{(1-z)^{2}} \prec \Psi(\alpha, \beta, \lambda ; z),
$$

implies

$$
\frac{1+z}{1-z} \prec\left(\frac{z f^{\prime}(z)}{f(z)}\right)^{\lambda}
$$

and $\frac{1+z}{1-z}$ is the best subordinant.

\section{SANDWICH THEOREMS}

By combining the results of subordination and superordination, we get the following "Sandwich theorems". 
Theorem 5.1 : Let $q_{1}$ and $q_{2}$ be convex univalent in $\Delta$ and satisfying (9) and (6) respectively.

Let $f \in \mathcal{A},\left(\frac{z f^{\prime}(z)}{f(z)}\right)^{\lambda} \in \mathcal{H}[1,1] \cap Q$ and $\Psi(\alpha, \beta, \lambda ; z)$ as defined by (7) is univalent in $\Delta$. Further if

$$
\alpha q_{1}(z)+\beta z q_{1}^{\prime}(z) \prec \Psi(\alpha, \beta, \lambda ; z) \prec \alpha q_{2}(z)+\beta q_{2}^{\prime}(z),
$$

then

$$
q_{1}(z) \prec\left(\frac{z f^{\prime}(z)}{f(z)}\right)^{\lambda} \prec q_{2}(z),
$$

and $q_{1}$ and $q_{2}$ are respectively the best subordinant and best dominant.

For $q_{1}(z)=\frac{1+A_{1} z}{1+B_{1} z}, q_{2}(z)=\frac{1+A_{2} z}{1+B_{2} z}\left(-1 \leq B_{2} \leq B_{1}<A_{1} \leq A_{2} \leq 1\right)$, we have the following Example.

Example 5.1 : If $f \in \mathcal{A},\left(\frac{z f^{\prime}(z)}{f(z)}\right)^{\lambda} \in \mathcal{H}[1,1] \cap Q$ and $\Psi(\alpha, \beta, \lambda ; z)$ as defined by (7) is univalent in $\Delta$, and

$$
\Psi_{1}\left(A_{1}, B_{1}, \alpha, \beta, \lambda ; z\right) \prec \Psi(\alpha, \beta, \lambda ; z) \prec \Psi_{2}\left(A_{2}, B_{2}, \alpha, \beta, \lambda ; z\right),
$$

then

$$
\frac{1+A_{1} z}{1+B_{1} z} \prec\left(\frac{z f^{\prime}(z)}{f(z)}\right)^{\lambda} \prec \frac{1+A_{2} z}{1+B_{2} z}
$$

where

$$
\begin{aligned}
& \Psi_{1}\left(A_{1}, B_{1}, \alpha, \beta, \lambda ; z\right):=\alpha\left(\frac{1+A_{1} z}{1+B_{1} z}\right)+\frac{\beta\left(A_{1}-B_{1}\right) z}{\left(1+B_{1} z\right)^{2}}, \\
& \Psi_{2}\left(A_{2}, B_{2}, \alpha, \beta, \lambda ; z\right):=\alpha\left(\frac{1+A_{2} z}{1+B_{2} z}\right)+\frac{\beta\left(A_{2}-B_{2}\right) z}{\left(1+B_{2} z\right)^{2}} .
\end{aligned}
$$

The functions $\frac{1+A_{1} z}{1+B_{1} z}$ and $\frac{1+A_{2} z}{1+B_{2} z}$ are respectively the best subordinant and best dominant.

\section{APPLICATION TO DZIOK-SRIVASTAVA OPERATOR}

Theorem 6.1 : Let $q$ be univalent in $\Delta$ with $q(0)=1$. Let $\eta(\alpha, \beta, \lambda, l, m ; z):=\left(\frac{H_{m}^{l}\left[\alpha_{1}+1\right] f(z)}{H_{m}^{l}\left[\alpha_{1}\right] f(z)}\right)^{\lambda} \times$

$$
\left[(\alpha+\beta \lambda)\left\{\frac{\left(\alpha_{1}+1\right)\left(H_{m}^{l}\left[\alpha_{1}+2\right] f(z)\right)}{H_{m}^{l}\left[\alpha_{1}+1\right] f(z)}-\alpha_{1}\left(\frac{H_{m}^{l}\left[\alpha_{1}+1\right] f(z)}{H_{m}^{l}\left[\alpha_{1}\right] f(z)}\right)-1\right\}\right] .
$$


If $f \in \mathcal{A}$ satisfies

$$
\eta(\alpha, \beta, \lambda, l, m ; z) \prec \alpha p(z)+\beta z q^{\prime}(z)
$$

then

$$
\left(\frac{H_{m}^{l}\left[\alpha_{1}+1\right] f(z)}{H_{m}^{l}\left[\alpha_{1}\right] f(z)}\right)^{\lambda} \prec q(z),
$$

and $q$ is the best dominant.

Proof. Define the function $p(z)$ by

$$
p(z):=\left(\frac{H_{m}^{l}\left[\alpha_{1}+1\right] f(z)}{H_{m}^{l}\left[\alpha_{1}\right] f(z)}\right)^{\lambda} .
$$

By taking logarithmic derivative of (11) we get

$$
\frac{z p^{\prime}(z)}{p(z)}=\lambda\left[\frac{z\left(H_{m}^{l}\left[\alpha_{1}+1\right] f(z)\right)^{\prime}}{H_{m}^{l}\left[\alpha_{1}+1\right] f(z)}-\frac{z\left(H_{m}^{l}\left[\alpha_{1}\right] f(z)\right)^{\prime}}{H_{m}^{l}\left[\alpha_{1}\right] f(z)}\right] .
$$

By using identity

$$
z\left(H_{m}^{l}\left[\alpha_{1}\right] f(z)\right)^{\prime}=\alpha_{1} H_{m}^{l}\left[\alpha_{1}+1\right] f(z)-\left(\alpha_{1}-1\right) H_{m}^{l}\left[\alpha_{1}\right] f(z),
$$

and (11) in (12) we get

$$
\begin{aligned}
& \alpha p(z)+\beta z p^{\prime}(z)=\left(\frac{H_{m}^{l}\left[\alpha_{1}+1\right] f(z)}{H_{m}^{l}\left[\alpha_{1}\right] f(z)}\right)^{\lambda} \times \\
& \quad\left[(\alpha+\beta \lambda)\left\{\frac{\left(\alpha_{1}+1\right) H_{m}^{l}\left[\alpha_{1}+2\right] f(z)}{H_{m}^{l}\left[\alpha_{1}+1\right] f(z)}-\alpha_{1}\left(\frac{H_{m}^{l}\left[\alpha_{1}+1\right] f(z)}{H_{m}^{l}\left[\alpha_{1}\right] f(z)}\right)-1\right\}\right] .
\end{aligned}
$$

Now Theorem 6.1 follows as an application of Lemma 2.1.

By taking $l=2, m=1$ and $\alpha_{2}=1$ in Theorem 6.1 we have the following corollary.

Corollary 6.1 : Let $q$ be univalent in $\Delta$ with $q(0)=1$. Let $\phi(a, c, \alpha, \beta, \lambda: z):=\left(\frac{L(a+1, c) f(z)}{L(a, c) f(z)}\right)^{\lambda} \times$

$$
\left[\alpha+\beta \lambda\left\{\frac{(a+1) L(a+2, c) f(z)}{L(a+1, c) f(z)}-\frac{a L(a+1, c) f(z)}{L(a, c) f(z)}-1\right\}\right] .
$$

If $f \in \mathcal{A}$ satisfies

$$
\phi(a, c, \alpha, \beta, \lambda: z) \prec \alpha q(z)+\beta z q^{\prime}(z),
$$

then

$$
\left(\frac{L(a+1, c) f(z)}{L(a, c) f(z)}\right)^{\lambda} \prec q(z)
$$

and $q$ is the best dominant. 
Taking $a=1$ and $c=1$ in corollary 6.1 we get the following corollary.

Corollary 6.2 : Let $q$ be univalent in $\Delta$ with $q(0)=1$. If $f \in \mathcal{A}$ and

$$
\left(\frac{D^{n+1} f(z)}{D^{n} f(z)}\right)^{\lambda}\left[\alpha+\beta \lambda\left\{\frac{(a+1) D^{n+2} f(z)}{D^{n+1} f(z)}-\frac{a D^{n+1} f(z)}{D^{n} f(z)}-1\right\}\right] \prec \alpha q(z)+\beta z q^{\prime}(z),
$$

then

$$
\left(\frac{D^{n+1} f(z)}{D^{n} f(z)}\right)^{\lambda} \prec q(z)
$$

and $q$ is the best dominant.

Since the superordination results are a dual of the subordination here we state only the results pertaining to the superordination.

Theorem 6.2 : Let $q$ be convex univalent in $\Delta$ with $q(0)=1$. Let $f \in \mathcal{A}$, $\left(\frac{H_{m}^{l}\left[\alpha_{1}+1\right] f(z)}{H_{m}^{l}\left[\alpha_{1}\right] f(z)}\right)^{\lambda} \in \mathcal{H}[1,1] \cap Q$, with $\Re\left[\frac{\alpha}{\beta} q^{\prime}(z)\right]>0$. Further if $\eta(\alpha, \beta, \lambda, l, m ; z)$ as defined by (10) is univalent in $\Delta$, with

$$
\alpha q(z)+\beta z q^{\prime}(z) \prec \eta(\alpha, \beta, \lambda, l, m ; z)
$$

then

$$
q(z) \prec\left(\frac{H_{m}^{l}\left[\alpha_{1}+1\right] f(z)}{H_{m}^{l}\left[\alpha_{1}\right] f(z)}\right)^{\lambda},
$$

and $q$ is the best subordinant.

Theorem 6.3 : Let $q$ be convex univalent in $\Delta$.

Let $f \in \mathcal{A},\left(\frac{L(a+1, c) f(z)}{L(a, c) f(z)}\right)^{\lambda} \in \mathcal{H}[1,1] \cap Q$ and $\phi(a, c, \alpha, \beta, \lambda: z)$ as defined by (13) is univalent in $\Delta$. If

$$
\alpha q(z)+\beta z q^{\prime}(z) \prec \phi(a, c, \alpha, \beta, \lambda: z)
$$

then

$$
q(z) \prec\left(\frac{L(a+1, c) f(z)}{L(a, c) f(z)}\right)^{\lambda}
$$

and $q$ is the best subordinant.

Taking $q(z)=\frac{1+A z}{1+B z}, \frac{1+z}{1-z}$ in Theorem 6.1 we can get more results and we omit the details involved.

Combining the results of subordination and superordination, we state the following Sandwich Theorems. 
Theorem 6.4 : Let $q_{1}$ and $q_{2}$ be convex univalent in $\Delta$ satisfying (9) and (6) respectively. If $f \in \mathcal{A},\left(\frac{H_{m}^{l}\left[\alpha_{1}+1\right] f(z)}{H_{m}^{l}\left[\alpha_{1}\right] f(z)}\right)^{\lambda} \in \mathcal{H}[1,1] \cap Q$ and $\eta(\alpha, \beta, \lambda, l, m ; z)$ as defined by (10) is univalent in $\Delta$, and

$$
\alpha q_{1}(z)+\beta z q_{1}^{\prime}(z) \prec \eta(\alpha, \beta, \lambda, l, m ; z) \prec \alpha q_{2}(z)+\beta z q_{2}^{\prime}(z),
$$

then

$$
q_{1}(z) \prec\left(\frac{H_{m}^{l}\left[\alpha_{1}+1\right] f(z)}{H_{m}^{l}\left[\alpha_{1}\right] f(z)}\right)^{\lambda} \prec q_{2}(z),
$$

and $q_{1}(z)$ and $q_{2}(z)$ are respectively the best subordinant and best dominant.

For $q_{1}(z)=\frac{1+A_{1} z}{1+B_{1} z}, q_{2}(z)=\frac{1+A_{2} z}{1+B_{2} z}\left(-1 \leq B_{2}<B_{1}<A_{1}<A_{2} \leq 1\right)$, we have the following corollary.

Corollary 6.3 : If $f \in \mathcal{A},\left(\frac{H_{m}^{l}\left[\alpha_{1}+1\right] f(z)}{H_{m}^{l}\left[\alpha_{1}\right] f(z)}\right)^{\lambda} \in \mathcal{H}[1,1] \cap Q$ and $\eta(\alpha, \beta, \lambda, l, m ; z)$ as defined by (10) is univalent in $\Delta$, and

$$
\Phi_{1}\left(A_{1}, B_{1}, \alpha, \beta ; z\right) \prec \eta(\alpha, \beta, \lambda, l, m ; z) \prec \Phi_{2}\left(A_{2}, B_{2}, \alpha, \beta ; z\right),
$$

where

$$
\begin{gathered}
\Phi_{1}\left(A_{1}, B_{1}, \alpha, \beta ; z\right):=\alpha\left(\frac{1+A_{1} z}{1+B_{1} z}\right)+\frac{\beta\left(A_{1}-B_{1}\right) z}{\left(1+B_{1} z\right)^{2}}, \\
\Phi_{2}\left(A_{2}, B_{2}, \alpha, \beta ; z\right):=\alpha\left(\frac{1+A_{1} z}{1+B_{1} z}\right)+\frac{\beta\left(A_{2}-B_{2}\right) z}{\left(1+B_{2} z\right)^{2}}, \\
\Rightarrow \quad \frac{1+A_{1} z}{1+B_{1} z} \prec\left(\frac{H_{m}^{l}\left[\alpha_{1}+1\right] f(z)}{H_{m}^{l}\left[\alpha_{1}\right] f(z)}\right)^{\lambda} \prec \frac{1+A_{2} z}{1+B_{2} z} .
\end{gathered}
$$

The functions $\frac{1+A_{1} z}{1+B_{1} z}$ and $\frac{1+A_{2} z}{1+B_{2} z}$ are respectively the best subordinant and best dominant.

Theorem 6.5 : Let $q_{1}$ and $q_{2}$ be convex univalent in $\Delta$ and satisfing (9) and (6) respectively. If $f \in \mathcal{A},\left(\frac{L(a+1, c) f(z)}{L(a, c) f(z)}\right)^{\lambda} \in \mathcal{H}[1,1] \cap Q$ and $\phi(a, c, \alpha, \beta, \lambda: z)$ as defined by (13) is univalent in $\Delta$, and

$$
\alpha q_{1}(z)+\beta z q_{1}^{\prime}(z) \prec \phi(a, c, \alpha, \beta, \lambda: z) \prec \alpha q_{2}(z)+\beta z q_{2}^{\prime}(z)
$$

then

$$
q_{1}(z) \prec\left(\frac{L(a+1, c) f(z)}{L(a, c) f(z)}\right)^{\lambda} \prec q_{2}(z)
$$

and $q_{1}(z)$ and $q_{2}(z)$ are respectively the best subordinant and best dominant. 
Theorem 6.6 : Let $q_{1}(z)$ and $q_{2}(z)$ be convex univalent in $\Delta$ and satisfing (9) and (6) respectively. Let $f \in \mathcal{A},\left(\frac{D^{n+1} f(z)}{D^{n} f(z)}\right)^{\lambda} \in \mathcal{H}[1,1] \cap Q$,

$$
\left(\frac{D^{n+1} f(z)}{D^{n} f(z)}\right)^{\lambda}\left[\alpha+\beta \lambda\left\{\frac{(a+1) D^{n+2} f(z)}{D^{n+1} f(z)}-\frac{a D^{n+1} f(z)}{D^{n} f(z)}-1\right\}\right]
$$

is univalent in $\Delta$. Further if

$$
\begin{aligned}
\alpha q_{1}(z)+\beta z q_{1}^{\prime}(z) \prec\left(\frac{D^{n+1} f(z)}{D^{n} f(z)}\right)^{\lambda} \times \\
\quad\left[\alpha+\beta \lambda\left\{\frac{(a+1) D^{n+2} f(z)}{D^{n+1} f(z)}-\frac{a D^{n+1} f(z)}{D^{n} f(z)}-1\right\}\right] \prec \alpha q_{2}(z)+\beta z q_{2}^{\prime}(z),
\end{aligned}
$$

then

$$
q_{1}(z) \prec\left(\frac{D^{n+1} f(z)}{D^{n} f(z)}\right)^{\lambda} \prec q_{2}(z),
$$

and $q_{1}$ and $q_{2}$ are respectively the best subordinant and best dominant.

\section{REFERENCES}

1. S.D. Bernard, "Convex and starlike univalent functions", Trans. Amer. Math. Soc. 135 (1969), 429-446.

2. T. BulboacA , "A class of superordination-preserving integral operators", Indag. Math. (N.S.) 13(3) (2002), 301-311.

3. T. BulboACĂ, "Classes of first-order differential superordinations", Demonstratio Math. 35(2) (2002), 287-292.

4. B.C. Carlson And D.B. Shaffer, "Starlike and prestarlike hypergeometric functions", SIAM J. Math. Anal. 15(4) (1984), 737-745.

5. J. Dziok and H.M. SRivastava, "Certain subclasses of analytic functions associated with the generalized hypergeometric function", Integral Transforms Spec. Funct. 14(1) (2003), 7-18.

6. Ju. E. Hohlov, "Operators and operations on the class of univalent functions", Izv. Vyssh. Uchebn. Zaved. Mat. 10(197) (1978), 83-89.

7. R.J. Libera, "Some classes of regular univalent functions", Proc. Amer. Math. Soc. 16 (1965), 755-758.

8. A.E. Livingston, "On the radius of univalence of certain analytic functions", Proc. Amer. Math. Soc. 17 (1966), 352-357.

9. S.S. Miller and P.T. Mocanu, Differential Subordinations: Theory and Applications. Monographs and Textbooks in Pure and Applied Mathematics 225, Marcel Dekker, New York, 2000.

10. S.S. Miller And P.T. Mocanu, "Subordinants of differential superordinations", Complex Var. Theory Appl. 48(10) (2003), 815-826. 
11. St. Ruscheweyh, "New criteria for univalent functions", Proc. Amer. Math. Soc. 49 (1975), 109-115.

12. T.N. Shanmugam, V. Ravichandran and S. Sivasubramanian, "Differential sandwich theorems for some subclasses of analytic functions", Austra. J. Math. Anal. Appl. 3(1), (2006), 1-11.

13. H.M. SRIVAStava, "Some families of fractional derivative and other linear operators associated with analytic, univalent, and multivalent functions", in Analysis and its applications (Chennai, 2000), 209-243, Allied Publ., New Delhi.

T.N. Shanmuguam: Department of Mathematics, Anna University, Chennai - 25, Tamilnadu, India

M.P. Jeyaraman: Department of Mathematics, Eswari Engineering College, Chennai 89, Tamilnadu, India

E-mail : jeyaraman-mp@yahoo.co.in

A. Singaravelu: Department of Mathematics, Valliammai Engineering College, Kattankolathur, Kancheepuram District, Tamilnadu, India.

E-mail : asing-59@yahoo.com 